\title{
El uso simbólico del territorio como elemento de una propuesta conceptual del turismo gastronómico. Metodología para el desarrollo de una ruta agro-gastronómica
}

The symbolic use of the land as a conceptual proposal of culinary tourism. A methodology for creating an agro-gastronomic tour

\section{Elizabeth Sara Gómez Castillo* \\ Frida Lina Morcia Rivera}

Escuela Profesional de Turismo y Hotelería,

Universidad de San Martín de Porres, Perú

\section{Resumen}

La tradición, los paisajes naturales, la biodiversidad y la cultura, son algunos de los recursos turísticos con los que cuentan las zonas rurales. Este patrimonio cultural, refleja la identidad de una sociedad, pues durante el tiempo han desarrollado formas de adaptación y evolución social, así como destrezas, saberes, tecnologías relacionadas con el uso de la tierra. Teniendo en cuenta que en los últimos años, los visitantes buscan experiencias significativas y socioemocionales, los espacios rurales son una oportunidad para vivir esas experiencias por medio de la gastronomía.

El objetivo de este estudio es presentar una propuesta conceptual del turismo gastronómico sobre la base del uso simbólico del territorio, y además, plantear una metodología viable para el desarrollo de una ruta agrogastronómica, como una iniciativa para diversificar la actividad turística, preservando las costumbres y tradiciones locales; y a la vez, ser un elemento impulsor de nuevos ingresos económicos en zonas rurales.

Palabras clave: gastronomía; turismo; ruta agro-gastronómica; cocina local; turismo gastronómico

Este es un artículo Open Access bajo la licencia Creative Commons Atribución-NoComercial-Compartirlgual 4.0

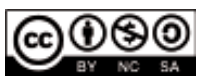




\begin{abstract}
Tradition, natural landscapes, biodiversity and culture are some of the tourism resources available in rural areas. This cultural heritage reflects the identity of a society because it has developed over time forms of adaptation and social evolution, as well as skills, knowledge and technologies related to land use. Bearing in mind that, in recent years, visitors seek significant and socio-emotional experiences, rural spaces are an opportunity to live those experiences through gastronomy.
\end{abstract}

This article aims to present a conceptual proposal of culinary tourism based on the symbolic use of the land and set out a viable methodology for creating an agro-gastronomic tour as an initiative to diversify tourism by preserving local customs and traditions, and as a driving force to generate new economic income in rural areas.

Keywords: gastronomy; tourism; agro-gastronomic tour; local cuisine; culinary tourism

\title{
Introducción
}

La evolución y los cambios que se dan en torno a la actividad turística, han originado la existencia de nuevas tendencias, se ha desarrollado un turismo más especializado con el fin de cubrir las expectativas del visitante. En ese sentido, la gastronomía se ha convertido en un recurso importante, dando apertura a posibilidades de desarrollo sobre la base del patrimonio cultural y culinario de una comunidad, exponiendo por medio de la cocina, la identidad, así como la riqueza del espacio natural y social.

El conocer una cultura, explorar, descubrir y degustar la culinaria de un determinado lugar, de acuerdo a lo observado, se está convirtiendo en una de las motivaciones principales de viaje. Debido a ello surgen las experiencias hands on, es decir, involucrarse en todo el proceso para la elaboración de un plato; desde visitar granjas o chacras, mercados, elegir y sentir el ingrediente local perfecto y cocinarlo uno mismo bajo la supervisión de un experto (Comisión de Promoción del Perú para la Exportación y el Turismo PromPerú, 2016). 
El presente estudio, aborda el turismo gastronómico en zonas rurales y el uso simbólico que se presenta en torno a ella como parte de su identidad. Hablar de cocina tradicional de un entorno rural, es hablar de saberes y tradiciones milenarias en el uso de la tierra, de conservación y técnicas en la preparación de alimentos, que han pasado de generación en generación; simbolismos que la caracterizan frente a otras culturas, representando de esta forma la vida del hombre en sociedad y su relación con la naturaleza.

En la construcción y planificación de rutas turísticas y gastronómicas, la metodología tradicional para el desarrollo de la actividad turística, ha tenido siempre en cuenta como punto de partida el espacio geográfico, conocido dentro del turismo como espacio turístico. El presente trabajo plantea, sin embargo, el uso de los símbolos que se dan en el territorio rural, como recurso gastronómico valioso para la creación de paisajes culturales y el desarrollo de una nueva metodología para la elaboración de rutas agro-gastronómicas, teniendo en cuenta las características propias del patrimonio culinario. «El patrimonio culinario es un elemento de comunicación cultural, y en ello se manifiestan tanto las tradiciones propiamente culturales como las idiosincrasias naturales de un lugar» (Fusté-Forne, 2016, p. 5). Esto refuerza el concepto de identidad y tradición, rescata la cultura local como baluarte para el turismo vivencial y la experiencia turística actual.

Por lo tanto, se empleó el diseño etnográfico del paradigma cualitativo de investigación social que permite conocer el contexto sociocultural del objeto de estudio (Hernández, Fernández, \& Baptista, 2014).

Durante su elaboración se usaron las siguientes técnicas: recolección de datos, observación, análisis de documentos y entrevista en profundidad a un experto. Se hizo contacto con el doctor Pedro Pablo Ccopa, sociólogo e investigador experto en temas migratorios, culinarios, entre otros -en el mes de agosto de 2019- para solicitar su opinión sobre el tema. Se diseñó un guión semiestructurado con los temas pertinentes, según la literatura revisada, que fue puesto a consideración del entrevistado quien se manifestó de acuerdo. Cabe señalar que la entrevista tuvo una duración de 40 minutos.

A continuación, se presenta el balance de las referencias conceptuales en las que se basa esta investigación. 


\section{El uso simbólico del territorio}

Toda actividad turística se desarrolla en un espacio geográfico. El uso del territorio implica prácticas económicas, socioculturales y ambientales, que pueden generar un impacto positivo o negativo en la comunidad receptora, así como en el visitante que se traslada para experimentar nuevas vivencias que le permitan sentir, disfrutar, recorrer y ubicarse por el valor simbólico del espacio cultural del destino visitado, acción que pone en valor el espacio territorial (Cammarata, 2006).

Al respecto, Rojas (2018) sostiene que la definición de geografía ha evolucionado en el tiempo, pasando del concepto de espacio geográfico hasta la relación sociedad-ambiente. La evolución del concepto de geografía como recurso material o espacio social, vinculándolo con las representaciones culturales con las que los pobladores rurales mantienen lazos de pertenencia y valores identitarios, hoy es posible gracias a la geografía positivista. Hablar de sensibilidad, de espacios vividos, apego a la tierra, identidad nacional, paisajes culturales, entre otros elementos, complementan el constructo primigenio de territorio; que mediante las experiencias y sensaciones, los pobladores locales interiorizan como referencia simbólica en su propio sistema cultural, apelando a la memoria colectiva vinculada a su historia y geografía.

Existen sabores que hacen que uno recuerde su infancia, sensaciones que están en la memoria, recuerdos adquiridos en torno a la mesa que dan nostalgia, así como también, hay perfumes que rememoran una preparación emblemática y característica de algún lugar. Zapata (s. f.) asevera que la forma más tenaz del recuerdo es el perfume, pues de todos nuestros sentidos el olfato puede despertar en el hombre nostalgia ya que guarda un alto valor emocional.

El perfume de la canela, por ejemplo, rememora la ciudad de Lima, es la especia que inspira una canción en su nombre, así como el sabor y aroma de los más tradicionales postres limeños. El perfume de las hierbas aromáticas nativas, cuya fragancia y sabor junto a las texturas de las carnes en platos festivos o ceremoniales como la pachamanca, evoca los Andes peruanos. El aroma y el sabor del limón, combinado con el ají y los frutos de mar, que nos recuerda a la costa peruana, son ejemplos de simbolismos que representan una cultura e identidad, permitiendo asociarlas con una determinada cocina. 
Por otro lado, Álvarez (1997) señala: «Qué sería del Perú, que sería del peruano si careciera de una memoria del olor, qué sería de nuestros sabores si no tuviéramos nuestros referentes, nuestros hitos que identifican a nuestras regiones» (p. 115). Todo ello es parte de la cultura definida como «el conjunto de componentes que conforman la identidad de un pueblo y que incluyen aspectos tan diversos como el territorio, el clima, la historia, la lengua, los símbolos, así como valores y normas» (Fusté-Forne, 2016, p. 6); elementos que se conjugan para maximizar la experiencia turística, cargada de simbolismos que forman parte del acervo cultural.

Y es que la gastronomía es la cultura de un pueblo puesta en la mesa, es el disfrutar sabores, colores, texturas que brindan la comida y bebida gracias a la memoria. Es el patrimonio intangible, expresión de un país, de una región que representa y da identidad; una expresión de arte culinario, que transmite sensaciones placenteras al paladar (Zapata, s. f.).

Para Fusté-Forne (2016) la gastronomía es considerada un símbolo territorial, es «una muestra tanto de la cultura como de la naturaleza que nos define como seres humanos con arraigo a un determinado lugar» (p. 6). Es por medio de los alimentos que existe una representación social de la cultura del hombre, así como de la biodiversidad de su territorio.

Por otra parte, Gómez (2018) afirma que la comida en zonas rurales cotidianamente cumple un significado simbólico, en la relación hombre y naturaleza; ello se refleja en las preparaciones de los alimentos, el uso de técnicas, insumos, utensilios a los cuales se agregan valores culturales, códigos sociales y simbolismos que representan al hombre alrededor de la producción, elaboración, deguste de la comida, así como todo lo concerniente a ella. Son aquellos elementos de la culinaria, legado que se hereda y transmite de generación en generación, como insumos, perfumes, texturas, que brindan sensación de placer al ser humano además de dar identidad.

Sobre el tema, el sociólogo e investigador especializado en cocinas regionales, Pedro Pablo Ccopa, refiere que desde el momento en que se otorga significado a las cosas se le da un valor simbólico: 
Cuando comemos, no solo comemos, sino que otorgamos significado a ese hecho. Por ello, por ejemplo, en el mundo prehispánico, la comida era parte de un ritual de agradecimiento a la tierra, a la pachamama, parte de la ofrenda a los dioses. Esta tradición ha sobrevivido a los embates de la modernidad en el mundo rural actual, en tanto el comer tiene aún ese significado, por ello el primer brindis de chicha es con la pachamama (2013).

Asimismo, Ccopa (2013) afirma que la comida es transversal, es decir no es exclusivo de un determinado lugar. Sin embargo, lo que lo distinguirá de otras cocinas son las formas de condimentación, por ejemplo, la forma en cómo se producen los insumos necesarios para la elaboración de la comida, es reconocer con qué insumos se cuenta, así como tener en cuenta el cómo se realiza cada preparación, ya que la cocina de un determinado lugar, tiene particularidades propias que identifican a un grupo social.

\section{El turismo gastronómico}

La actividad turística en el mundo ha ido evolucionando con el paso del tiempo, las diversas tendencias actuales han modificado los gustos y preferencias del visitante. La oferta turística ya no es exclusiva del sol y playa, han surgido nuevos destinos con el fin de ofrecer el disfrute a plenitud de la experiencia turística. El turismo gastronómico es uno de ellos. Schlüter (2003) al respecto, señala que el turismo gastronómico puede ser distinguido como una forma de turismo de interés específico, que surge a causa de la evolución social.

La gastronomía ya no solo es considerada como parte del itinerario de viaje, en donde el turista se alimenta solo por necesidad; ahora está logrando movilizar un número considerable de visitantes, interesados en descubrir la cultura de un país mediante la degustación y disfrute de la comida local, el hecho de conocer los insumos, los procesos y todo lo referente a ella. ${ }^{1}$

Cabe señalar que el estudio Evaluación del Turismo Gastronómico en el Perú, realizado por Promperú (2016), ha considerado como universo a vacacionistas extranjeros mayores de 18 años provenientes de los siguientes países: EE.UU., Francia, Colombia, Brasil, Argentina y España. 
Sobre el concepto, los especialistas Hall y Sharples (2003) refieren de turismo gastronómico:

La visita a productores primarios y secundarios de alimentos, festivales gastronómicos, restaurantes y lugares específicos donde la degustación de platos y/o la experimentación de los atributos de una región especializada en la producción de alimentos es la razón principal para la realización de un viaje. (p. 10)

Asimismo, el turismo gastronómico no solo es importante dentro de la experiencia turística, sino que es un elemento clave de identidad para las sociedades postmodernas.

Por otro lado, Morcia (2013) señala: «Es importante recordar que entre los principales objetivos del turismo es el conocer otros pueblos y culturas, siendo la gastronomía, un objetivo turístico al formar parte de esta cultura». Tomándose en cuenta que dentro del folclore, la gastronomía ocupa un lugar relevante como parte de la preservación de la identidad de un pueblo.

Mientras que Millán y Agudo (2010) sostienen que el turismo gastronómico se da cuando el principal motivo de movilización son las visitas a productores primarios y secundarios de alimentos, a restaurantes, a festivales gastronómicos y todo lugar donde el visitante puede degustar la comida local.

Con relación al turismo gastronómico y el entorno rural, Fusté-Forne (2016) afirma que existe una estrecha relación entre gastronomía, vida rural y el sector servicios, ya que al tener en cuenta a la cocina como recurso, se pueden crear diversos paisajes que dan lugar al desarrollo local y turístico, siendo la gastronomía ese «símbolo territorial» pues las prácticas en torno a ella, son la materialización de aquellos saberes y sabores que guarda una cultura durante el tiempo, da a conocer la riqueza natural que posee, su cultura, manifiesta por medio de las tradiciones, así como la identidad de una determinada sociedad.

Respecto al perfil de los visitantes que hacen turismo gastronómico, Montecinos (2016) plantea la siguiente definición: 
Personas que durante sus viajes y estancias realizan actividades fundamentadas en el patrimonio cultural gastronómico inmaterial y material en diferentes lugares al de su entorno habitual, por un periodo de tiempo consecutivo inferior a un año con el principal objetivo de consumir y disfrutar los productos, servicios y experiencias gastronómicas de manera prioritaria y complementaria. (p. 98)

Montecinos (2016), investigador de temas gastronómicos, propone que para considerar a la gastronomía como atractivo gastronómico es importante diferenciarla como patrimonio tangible e intangible (ver Tabla 1).

\section{Tabla 1}

Propuesta para distinguir el patrimonio cultural gastronómico.

\begin{tabular}{ll}
\hline \multicolumn{1}{c}{ Patrimonio cultural gastronómico } \\
\hline \multicolumn{1}{c}{ Patrimonio tangible } & \multicolumn{1}{c}{ Patrimonio intangible } \\
\hline - Actividades agrarias: pesca, siembra, & - Recetas orales \\
$\begin{array}{l}\text { cosecha con técnicas de cultivos, } \\
\text { procedimientos y preparación culinaria }\end{array}$ & - Técnicas culinarias \\
únicos en su género. & - Conocimientos prácticos ancestrales, \\
- Prácticas rituales de cocina cotidiana, & relacionados con la naturaleza, el universo, \\
festiva y ceremonial. & técnicas culinarias. \\
- Preparación culinaria con uso de & Costumbres y métodos de comportamiento \\
utensilios especiales para la degustación & comunitario ancestrales. \\
de los manjares. & Elementos culturales que propician la interacción \\
- Bebidas tradicionales & social y la armonía entre las personas. \\
- Los productos de la naturaleza & Expresiones de identidad comunitaria: actos \\
- Artesanías relacionadas con la & festivos, que fortalecen vínculos sociales y \\
gastronomía. & consolidan el sentimiento de identidad a nivel \\
& local, regional y nacional. \\
& Práctica viva de los rituales gastronómicos y la \\
& transmisión para su preservación oral o escrita \\
& por las nuevas generaciones.
\end{tabular}

Fuente: Montecinos, 2016.

En consecuencia, se puede sostener que el turismo gastronómico es la movilización de personas que buscan conocer el patrimonio cultural de una sociedad mediante su cocina. Es el interés del visitante por salir de su entorno habitual, con el objetivo de buscar experiencias que le permitan generar vínculos interculturales, por medio del disfrute de vivencias relacionadas con 
el patrimonio culinario material e inmaterial durante la participación activa en diversas labores, propias del anfitrión, logrando así descubrir y ser parte de costumbres, saberes e historia; degustar perfumes y sabores, todo lo que gira en torno a la alimentación en un espacio rural.

\section{El turismo en el espacio rural: agroturismo como agente de desarrollo}

De acuerdo a Riveros y Blanco (2003) la actividad turística tiene distintas modalidades y el agroturismo es una de ellas, puesto que permite al visitante conocer la cultura local y experimentar de manera práctica la realización de actividades cotidianas vinculadas a la tierra, como el cultivo ancestral, las formas de cosecha, así como la elaboración de productos agroalimentarios, como por ejemplo, el queso o la mantequilla. También, les permite participar en la realización de artesanía y otras actividades que forman parte de la comunidad.

Asimismo, los autores señalan que estas actividades se realizan en fincas o chacras donde el visitante puede descansar y disfrutar del paisaje rural, compartiendo el día a día con el productor y sus familias.

Vidal y Mota (2017), afirman que es una alternativa para que la comunidad receptora obtenga beneficios y aproveche los recursos naturales que posee, permitiendo la conservación y preservación de estos recursos, y con este fin, la zona se pueda convertir en un medio sostenible para los actores involucrados en el desarrollo de esta actividad. Aseveran además, que la clave radica en brindar un buen servicio en un área natural ofreciendo productos locales.

Para Ramos (2018), el agroturismo involucra la participación y exposición, de los diferentes procesos agrícolas que se dan en las comunidades campesinas, las actividades cotidianas del productor, así como en la alimentación propia del lugar. También se consideran la convivencia y comunicación directa entre anfitrión y visitante.

El agroturismo, es por lo tanto, una modalidad del turismo rural, que involucra de forma cercana al anfitrión y al visitante mediante la exposición participativa de las diferentes actividades agropecuarias y/o agroindustriales, 
que se producen diariamente; así como en la preparación y disfrute de alimentos. Esta interacción cercana, permite al visitante identificar la biodiversidad que posee el entorno visitado y aprender sobre la tecnología, saberes y la cultura del mundo rural.

Es importante tener en cuenta que mediante las actividades agroturísticas, es posible diversificar las actividades agrícolas y agroindustriales, beneficiando de manera directa e indirecta no solo a empresarios sino también a otras comunidades que, gracias al desarrollo de actividades turísticas, tendrán la posibilidad de generar ingresos para sus familias y mejorar así su calidad de vida, permaneciendo en sus chacras y fortaleciendo su identidad, sintiéndose orgullosos de su entorno rural y de la valoración que los visitantes le dan a este.

\section{Propuesta metodológica para el desarrollo de una ruta agro- gastronómica}

En el Manual para la planificación de productos turísticos del Ministerio de Comercio Exterior y Turismo de Perú - MINCETUR (2014) se detalla que todo producto turístico consta de tres elementos: material, que es lo que está ligado al producto turístico (infraestructura, recursos naturales); experiencial, que es lo concerniente a la participación de eventos, actividades de esparcimiento, festividades, vínculos con los anfitriones del destino, calidad del servicio, además de la seguridad y tranquilidad; emocional, factor humano y cultural, así como la hospitalidad de los anfitriones del destino.

Por otro lado, Di Clemente (2016), sostiene que las experiencias y los componentes emocionales, son al parecer las nuevas tendencias de la demanda turística, posicionando así al turismo gastronómico entre las opciones más atractivas a la hora de hacer turismo; pues permiten participar en la prestación de servicios, conocer nuevas culturas alimentarias y posibilitan, además, disfrutar experiencias sensoriales placenteras, trasmitiendo emociones y sentimientos mediante los sentidos.

El turismo gastronómico va más allá del solo hecho de visitar un espacio turístico y probar la culinaria local, ya que está relacionado con la búsqueda de experiencias, es el deseo de conocer, experimentar, descubrir sabores, 
perfumes, texturas, colores y todo relacionado con la cultura y la identidad de la gastronomía local.

El turista que viaja a un destino turístico no realiza meramente un viaje físico. El verdadero viaje es interior, y radica en cómo percibe el lugar que visita, sus gentes, y el impacto que esta percepción subjetiva y personal le produce. (Carballo, Moreno-Gil, León, \& Brent, 2015, p. 72)

Está en el hecho simbólico que refiere cada preparación de comida, desde el proceso de tratar la tierra para la siembra, hasta la degustación final del plato; todo ello muestra la estrecha relación entre territorio, cultura, identidad y alimentación. «La cocina habla de la experiencia de un pueblo, experiencia que no se concreta al momento, sino a experiencias de años, de sus variaciones a lo largo de la historia» (Ccopa, 2013, p. 34).

Es importante tener en cuenta que la gastronomía en el entorno rural, es una cocina tradicional, ya que entre sus características se puede identificar lo siguiente: son preparaciones heredadas de generación a generación, tienen antecedentes ancestrales, los productos usados para la elaboración son de su entorno, y su preparación y consumo son vigentes (Pazos, 2011). Otra de las características de la cocina en zonas rurales es la relación cercana del hombre con la naturaleza.

Teniendo en cuenta que los visitantes «no se satisfacen fácilmente con un evento artificial, sino que buscan la autenticidad de las experiencias» (Carballo et al., 2015, p. 73) se construye el objetivo del presente estudio, considerando el uso simbólico del territorio como uno de los elementos fundamentales de una cultura y la creación de toda la experiencia turística, pues la importancia de este patrimonio cultural inmaterial, «no estriba en la manifestación cultural en sí, sino en el acervo de conocimientos y técnicas que se transmiten de generación en generación» (Organización de las Naciones Unidas para la Educación, la Ciencia y la Cultura - UNESCO, 2011) factor importante de la diversidad cultural frente al mundo global. 


\section{Definición}

Con la finalidad de poder crear experiencias memorables en el recuerdo de los visitantes mediante la gastronomía en zonas rurales, se propone la elaboración de rutas basadas en experiencias. Debido a esto, se presenta la siguiente propuesta de definición:

Una ruta agro-gastronómica es un conjunto de actividades estructuradas con base en experiencias sensoriales vinculadas a la cocina auténtica y tradicional de zonas rurales, cumpliendo además con un itinerario basado en el patrimonio cultural-gastronómico. El uso de rutas gastronómicas es considerado como instrumento de salvaguarda de la cocina tradicional y expresión de su identidad.

Los objetivos que tiene la ruta agro-gastronómica propuesta son:

1. Diversificar la oferta turística tradicional.

2. Otorgar un rol protagónico a las características organolépticas que poseen los insumos utilizados en la cocina del campo; perfume, textura, sonidos, que en su sencillez y cotidianidad otorgan rasgos auténticos, además del reconocimiento de su biodiversidad.

3. Dar a conocer las costumbres y tradiciones en zonas rurales.

4. Preservar los usos y costumbres vinculadas a la preparación de los alimentos y todo lo que involucra la culinaria de cada pueblo.

\section{Lineamientos para elaborar una ruta agro-gastronómica}

Para Alemán (2014) es importante generar dentro de las prácticas turísticas estrategias interpretativas, teniendo en cuenta el extenso patrimonio cultural y natural que tiene el Perú:

Los turistas han tomado conciencia acerca del valor del patrimonio cultural y natural que los rodea, ya no se trata de acumular información, la cual puede ser obtenida en diversas fuentes y a gran velocidad, sino de comprender, de interiorizar, de vivir una experiencia que es en todo sentido personal y subjetiva. (p. 306) 
Por lo tanto, con el fin de proponer una metodología que permita el desarrollo de una ruta agro-gastronómica basada en experiencias sensoriales memorables, se formulan los siguientes lineamientos:

- Elaborar un registro del patrimonio culinario material e inmaterial y recursos turísticos de la zona rural a trabajar.

- Clasificar el patrimonio culinario material e inmaterial y los recursos turísticos.

- Reconocer los elementos que conforman el patrimonio culinario con potencial para experiencias sensoriales. Buscar el hilo conductual, por ejemplo, el perfume de una hierba aromática local usada en la preparación de una comida emblemática de la zona de estudio.

- Seleccionar el patrimonio culinario material e inmaterial (recursos gastronómicos) que pueden ser atractivos para la ruta.

- Reconocer las costumbres y tradiciones en el entorno rural. Por ejemplo: en el comer está el compartir, los frutos de la pachamama se tienen que compartir.

- Describir el patrimonio gastronómico (recurso gastronómico).

- Incorporar dentro del recorrido un laboratorio de experiencias sensoriales, basado en las características propias de los insumos locales. Se trabajaría con grupos pequeños, en un ambiente apropiado, sin estímulos externos que distorsionen la experiencia sensorial con los insumos elegidos.

- Medir el tiempo que tomará la ruta.

- Identificar la infraestructura turística; la que facilitará el recorrido de la ruta (ver Figura 1). 


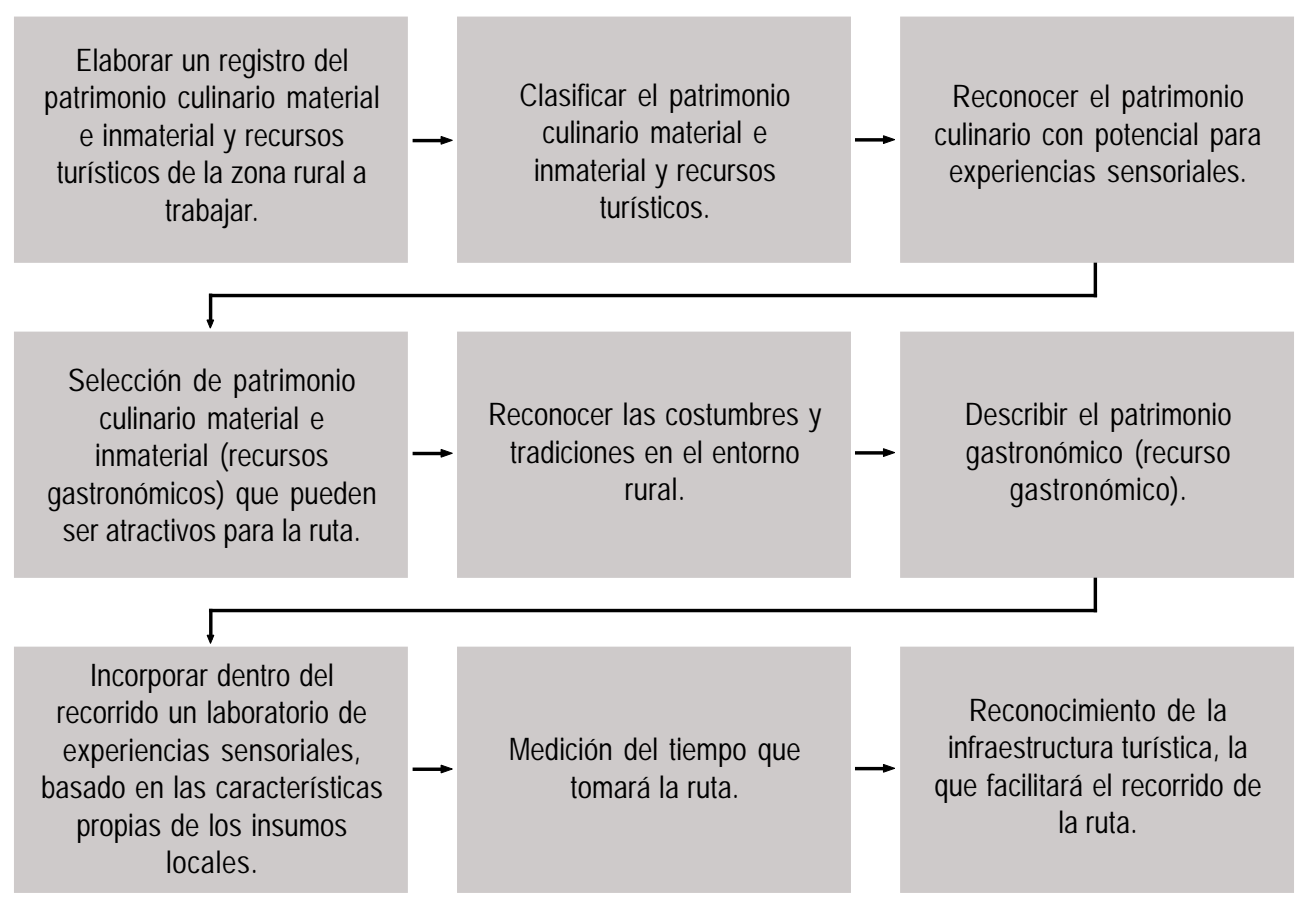

Figura 1. Propuesta metodología para el desarrollo de una ruta agro-gastronómica.

\section{Conclusiones}

El patrimonio gastronómico, independientemente del uso turístico, está siendo motivo principal para el desplazamiento de visitantes que buscan experiencias turísticas auténticas mediante la gastronomía. El uso simbólico del territorio, permite diversificar la oferta turística.

En este estudio se desmitifica el concepto tradicional de una ruta gastronómica que se basa en el uso del territorio, ya que se considera que el espacio geográfico es un elemento para el desplazamiento físico de los visitantes. Por otro lado, presentar una ruta gastronómica basada en los sentidos y las características organolépticas de los insumos utilizados en las comidas rurales $y$, que a su vez, pueda considerar los tres elementos, experiencial, atractivos y emociones para elaborar un producto turístico, harán sostenible esta propuesta a lo largo del tiempo, beneficiando no solo a los visitantes con la riqueza cultural y experiencial, sino también al poblador local; el cual tendrá un factor impulsor de su económica y, a la vez, preservará sus tradiciones. 
Se debe aprovechar la etapa de introducción a nivel mundial que está experimentando la cocina peruana como una de las mejores gastronomías del mundo, para seguir mostrando, por medio de esta expresión cultural, la construcción identitaria regional, como base de la gran cocina peruana y los paisajes naturales que el Perú posee.

\section{Referencias}

Alemán, A. (2014). Reflexiones sobre la interpretación del patrimonio. Cultura, 28, 305-315. Lima: Asociación de Docentes de la Universidad de San Martín de Porres.

Álvarez, I. (1997). Huellas y Sabores del Perú. Lima: Universidad de San Martín de Porres.

Cammarata, E. (2006). El turismo como práctica social y su papel en la apropiación y consolidación del territorio. En América Latina: cidade, campo e turismo (pp. 351-366). San Pablo: CLACSO, Consejo Latinoamericano de Ciencias Sociales. Recuperado de http://bibliotecavirtual.clacso.org.ar/ar/libros/edicion/lemos/ 20cammar.pdf

Carballo, R., Moreno-Gil, S., León, C., \& Brent, J. R. (2015). La creación y promoción de experiencias en un destino turístico. Un análisis de la investigación y necesidades de actuación. Cuadernos De Turismo, 35, 71-94. https://doi.org/10.6018/turismo.35.221511

Ccopa, P. (2013). Apurímac, cocina de runas y de dioses. En I. Álvarez (Dir.), Reconociendo y revalorando las cocinas regionales del Perú. Lima: Universidad de San Martín de Porres.

Comisión de Promoción del Perú para la Exportación y el Turismo - PromPerú. (2016). Turismo Gastronómico en el Perú. Recuperado de https://www.promperu.gob.pe/turismoin/Sitio/VisorDocumentos?titulo=Turismo\% 20Gastron\%C3\%B3mico\%20en\%20el\%20Per\%C3\%BA\&url= /Uploads/infografias/1048/ TurismoGastronomicoPeru2016.pdf\&nombObjeto=Infograf\%C3\%ADas

Di Clemente, E. (2016). Las variables experienciales como determinantes de la calidad de vida, la satisfacción y la lealtad del turista en el contexto del Turismo Gastronómico. Recuperado de https://dialnet.unirioja.es/ servlet/tesis?codigo $=66071$

Fusté-Forne, F. (2016). Los paisajes de la cultura: la gastronomía y el patrimonio culinario. Dixit, 24(1), 4-16. Recuperado de http://www.scielo.edu.uy/scielo.php?script=sci_arttext\&pid=S0797-36912016000100001

Gómez, E. (2018). Revalorización de la cocina tradicional del distrito de Anco Huallo (Uripa), en Chincheros Apurímac; a través de un registro gastronómico, para su difusión y desarrollo turístico (Tesis de maestría). Lima: Universidad de San Martín de Porres. Recuperado de http://www.repositorioacademico.usmp.edu.pe/ handle/usmp/4368

Hall, M., \& Sharples, L. (2003). The consumption of experiences or the experience of consumption? An introduction to the tourism of taste. Food Tourism Around the World, 1-24. doi: 10.1016/B978-0-7506-55033.50004-X

Hernández, R., Fernández, C., \& Baptista, P. (2014). Metodología de la investigación (6ª ed.). Ciudad de México: McGraw Hill.

Millán, G., \& Agudo, E. (2010). El Turismo gastronómico y las denominaciones de origen en el sur de España: Oleoturismo un estudio de caso. Pasos: Revista de Turismo y Patrimonio Cultural, 8(1), 91-112. Recuperado de http://www.redalyc.org/articulo.oa?id=88112836008 
Ministerio de Comercio Exterior y Turismo - MINCETUR. (2014). Manual de Planificación de Productos Turísticos. Recuperado de https://www.mincetur.gob.pe/wp-content/uploads/documentos/turismo/ consultorias/directoriosManuales/Manual-Planificacion-ProductosTuristicos-2014.pdf

Montecinos, A. (2016). Turismo Gastronómico Sostenible: Planificación de servicios, restaurantes, rutas, productos y destinos. México: Centro Empresarial Gastronómico Hotelero.

Morcia, F. (2013). La Cocina de Pozuzo como eje central de una ruta gastronómica para su desarrollo Turístico. (Tesis de maestría). Lima: Universidad de San Martín de Porres.

Organización de las Naciones Unidas para la Educación, la Ciencia y la Cultura - UNESCO. (2011). ¿Qué es el patrimonio cultural inmaterial? Recuperado de http://www.unesco.org/culture/ich/es/que-es-el-patrimonioinmaterial-00003

Pazos, S. (2011). Permanencias culturales y culinarias ecuatorianas en el manual de cocina de Juan Pablo Sanz (Quito, 1850-1860) y en el tratado de cocina de Adolfo Gehin (Quito, 1897). Recuperado de https:/l www.unibe.edu.ec/wp-content/uploads/2017/08/2.1permanencias-culturales-y-culinarias.pdf

Ramos, F. (2018). Huánuco: Geografía y Turismo Rural Comunitario (1ª ed.). Lima: Grandes Ediciones.

Riveros, H., \& Blancos, M. (2003). El agroturismo, una alternativa para revalorizar la agroindustria rural como mecanismo de desarrollo local. Recuperado de http://fediap.com.ar/administracion/pdfs/Agroturismo\%20\%20Una\%20alternativa\%20para\%20revalorizar\%20la\%20Agroindustria\%20Rural\%20(Hernando\%20Riveros).pdf

Schlüter, R. (2003). Turismo y Patrimonio gastronómico, una perspectiva ( $1^{\mathrm{a}}$ ed.). Buenos Aires: Centro de Investigaciones y Estudios Turísticos (CIET).

Vidal, E., \& Mota, S. (2017). El agroturismo y como se relaciona con el desarrollo turístico en el distrito de Huayllabamba, provincia de Urubamba, en el departamento de Cusco. LOGOS 7(1), 1-6. http://dx.doi.org/ 10.21503/log.v7i1.1424

Zapata, S. (s. f.). Principios del sabor en la gastronomía peruana. Recuperado de http://biblioteca.herdez.com.mx/ pdf/Principios.pd 